\author{
Yunchao Xie ${ }^{1}$ \\ Fei Chang ${ }^{1}$ \\ Chenlu $\mathrm{Li}^{1}$ \\ Juan Chen ${ }^{1}$ \\ Jieru Luo ${ }^{1}$ \\ Liang $\mathrm{Li}^{1}$ \\ Xuefeng $\mathrm{Hu}^{2}$ \\ ${ }^{1}$ School of Environment and \\ Architecture, University of Shanghai \\ for Science and Technology, \\ Shanghai, P. R. China \\ ${ }^{2}$ Key Laboratory of Coastal Zone \\ Environmental Processes, Yantai \\ Institute of Coastal Zone Research, \\ Chinese Academy of Sciences, \\ Yantai, P. R. China
}

\section{Research Article \\ One-Pot Polyvinyl Alcohol-Assisted Hydrothermal Synthesis of Hierarchical Flower-Like BiOCl Nanoplates with Enhancement of Photocatalytic Activity for Degradation of Rhodamine B}

\begin{abstract}
We have successfully synthesized hierarchical flower-like bismuth oxychloride nanoplates by a polyvinyl alcohol-assisted hydrothermal process. The as-synthesized products were characterized by a collection of techniques, such as X-ray diffraction, scanning electron microscopy, UV-Vis diffuse reflectance spectroscopy, and $\mathrm{N}_{2}$ adsorption-desorption isotherms. The concentration of polyvinyl alcohol and hydrothermal time were vital effects for formation of hierarchical flower-like bismuth oxychloride nanostructures. Rhodamine B $(\mathrm{RhB})$ was selected to evaluate the photocatalytic performance of as-synthesized samples under visible light irradiation. The degradation was undergone through a photosensitization pathway. The hierarchical flower-like bismuth oxychloride nanoplates showed much higher RhB removal efficiency than other bismuth oxychloride samples and commercial Degussa $\mathrm{TiO}_{2}$. The active species trapping experiments revealed that the superoxide radicals $\left({ }^{\bullet} \mathrm{O}_{2}^{-}\right)$were the main active species in aqueous solution. In combining with the $\mathrm{N}_{2}$ bubbling experiment, photocatalysis mechanism was finally proposed.
\end{abstract}

Keywords: Active species; Bismuth oxychloride; Dye; Photocatalysis

Received: January 7, 2013; revised: March 29, 2013; accepted: April 3, 2013

DOI: $10.1002 /$ clen.201300014

Additional supporting information may be found in the online version of this article at the publisher's web-site

\section{Introduction}

Heterogeneous photocatalysis is an attractive technology for control of environmental pollution and has become one of the most active research fields in recent years since it is able to completely mineralize organic pollutants under mild conditions $[1,2]$. As an important V-VI-VII ternary compound, bismuth oxychloride (BiOCl) exhibits a layer structure characterized by $\left[\mathrm{Bi}_{2} \mathrm{O}_{2}\right]$ slabs interleaved with double slabs of $\mathrm{Cl}$ atoms in the tetragonal matlockite structure $[3,4]$. The strong internal electric field between $\left[\mathrm{Bi}_{2} \mathrm{O}_{2}\right]$ slabs and $\mathrm{Cl}$ slabs facilitates an efficient separation of photoinduced electron-hole pairs [5-7]. Moreover, BiOCl belongs to the family of indirect band-gap semiconductors, featured with relatively slow recombination rate of photoinduced electrons and holes [3]. Both merits of $\mathrm{BiOCl}$ in structure are advantageous over other semiconductors for photocatalysis techniques. Therefore, $\mathrm{BiOCl}$ has been demonstrated as a promising and effective photocatalyst in decomposing organic pollutants $[8,9]$. To improve the photocatalytic performance, it is

Correspondence: Dr. F. Chang, School of Environment and Architecture, University of Shanghai for Science and Technology, Shanghai, 200093, P. R. China

E-mail: feichang@usst.edu.cn

Abbreviations: BiOCl, bismuth oxychloride; BQ 1,4-benzoquinone; EDX, energy-dispersive X-ray spectroscopy; $\mathbf{h}^{+}$, holes; IPA, isopropanol alcohol; PVA, polyvinyl alcohol; RhB, rhodamine B; SEM, scanning electron microscopy; TA, terephthalic acid; TOC, total organic carbon; UV-Vis DRS, UV-Vis diffuse reflectance spectroscopy; XRD, X-ray diffraction of great significance to design and prepare $\mathrm{BiOCl}$ nano/microstructures with ideal and controllable morphologies, dimensions, and sizes.

A vast array of synthetic procedures have been developed, such as template-aided synthesis [8,10], hydro/solvothermal preparation [11], sonochemical route [12], and ionothermal synthesis [13], to construct two dimensional (2D) BiOCl nanostructures of nanobelts [14, 15], nanofibers [15], nanoplates [13, 16], nanosheets [12, 17], and nanoflakes $[13,18]$. Especially, nanoplates are extensively studied not only for their unique optical and catalytic properties, but also for the applicable use to further fabricate 3D hierarchical nanoflowerlike or nanosphere-like structures [9]. Comparing to 2D nanostructures, 3D hierarchical nanoflower-like or nanospheric structures with features of large specific area values, strong light-harvesting ability, and easy recovery are capable of excellent photocatalytic performance $[19,20]$. Efficient synthesis of 2D nanoplates or 3D hierarchical nanostructures can be accomplished via a templateassisted preparation $[9,10]$. Chen reported that square-like $2 \mathrm{D} \mathrm{BiOCl}$ nanoplates by a mannitol-assisted hydrothermal synthesis exhibited excellent photocatalytic performance [6]. Yu observed that 3D hierarchical structured BiOCl exhibited higher photocatalytic capability than common BiOCl [8]. Zhang prepared BiOCl nanoflowers with pyridine and these samples showed excellent photocatalytic efficiency [9]. Zhang reported that 3D hierarchical BiOCl compounds synthesized through a PVP-assisted sonochemical route could effectively improve photocatalytic activity [10]. Xie found that 3D hierarchitectured BiOCl synthesized using a template-aided 
solvothermal method showed satisfactory photocatalytic activity [21]. Poly(vinyl alcohol) (PVA), a water-soluble polymer, contains carbon chain backbone with hydroxyl groups attached and facilitates the stabilization of metal ions by both means of chelation of cations with hydroxyl groups and physical entrapment of metal ions into the polymeric network [22]. The decrease of free metal ions in solution causes slow formation rate of nuclei and thus formation of crystals in high quality. In addition, the PVA polymer with bunch of hydroxyl groups on soft long carbon chains tends to combine separated nanostructures to form unique hierarchical morphologies.

The xanthene dye rhodamine $\mathrm{B}(\mathrm{RhB})$, is widely used as colorant in textiles and food production. If swallowed by human beings and animals, it is harmful to the skin, eyes and respiratory tract [23]. Therefore, it is important to remediate and eliminate $\mathrm{RhB}$ pollutants from water bodies. In this investigation, synthesis of $\mathrm{BiOCl}$ nanoplates with hierarchical flower-like morphology was first reported through a PVA-assisted hydrothermal route. A possible structure formation mechanism was also proposed. The photocatalytic performance of as-synthesized samples over RhB was conducted. The photocatalytic measurement indicated that $\mathrm{BiOCl}$ nanoplates synthesized with PVA exhibited much higher photocatalytic efficiency than BiOCl nanoflakes synthesized without PVA. Finally, the photocatalysis mechanism of BiOCl nanostructures was also carried out by active species trapping experiments.

\section{Materials and methods}

\subsection{Chemicals}

Bismuth nitrate pentahydrate $\left(\mathrm{Bi}\left(\mathrm{NO}_{3}\right)_{3} \cdot 5 \mathrm{H}_{2} \mathrm{O}, \mathrm{AR}\right)$, sodium chloride (NaCl, AR), PVA (AH-26), RhB (AR), isopropanol alcohol (IPA, AR), 1,4benzoquinone (BQ $\mathrm{CP})$, oxalic acid (AR), and terephthalic acid (TA, AR) were purchased from Sinopharm (Shanghai, China). Degussa $\mathrm{TiO}_{2}$ (P25, AR) was supplied by Degussa. All reagents were used without any further purification and all aqueous solutions were prepared with deionized water.

\subsection{Preparation of nanostructured $\mathrm{BiOCl}$ samples}

In a typical procedure, $3.0 \mathrm{~g} \mathrm{Bi}\left(\mathrm{NO}_{3}\right)_{3} \cdot 5 \mathrm{H}_{2} \mathrm{O}$ were given into a $60 \mathrm{~mL}$ PVA aqueous solution with different PVA concentrations. The suspension was ultrasonicated until the bismuth precursor was completely dissolved. The resultant mixture was allowed to stir for $1 \mathrm{~h}$ before an appropriate volume of saturated $\mathrm{NaCl}$ aqueous was added, resulting into the formation of a uniform white suspension. Afterward, this suspension was stirred for another $0.5 \mathrm{~h}$ and was then poured into a $100 \mathrm{~mL}$ stainless-steel Teflon-lined autoclave to undergo a hydrothermal process at $120^{\circ} \mathrm{C}$ for a desired reaction time. After naturally cooling down, the suspension was centrifuged, washed with distilled water and absolute ethanol several times to remove any soluble reactants and impurities, and collected as an off-white solid product. The obtained solid product were dried at $100^{\circ} \mathrm{C}$ overnight and calcined at $500^{\circ} \mathrm{C}$ for $4 \mathrm{~h}$ to supply the target $\mathrm{BiOCl}$ samples, denoting as $\mathrm{BOC}_{\mathrm{x}-\mathrm{y}}$, where $x$ and $y$ referred to the concentration of PVA $(\mathrm{g} / \mathrm{L})$ in aqueous and the hydrothermal time $(\mathrm{h})$, respectively.

\subsection{Characterization}

X-ray diffraction $(\mathrm{XRD})$ was carried out on a Bruker D8 Advance X-ray diffractometer using $\mathrm{Cu} \mathrm{K} \alpha$ radiation source $(\lambda=1.05406 \AA)$. The general morphology of as-prepared $\mathrm{BiOCl}$ samples was characterized by means of scanning electron microscopy (SEM, Hitachi S-4800). Energydispersive X-ray spectroscopy (EDX) was performed on an X-ray energy dispersive spectrometer Link 300 in connection with a SEM Philips XL 30. The optical property of the samples was measured through UV-Vis diffuse reflectance spectroscopy (UV-Vis DRS, Hitachi U-4100) using $\mathrm{BaSO}_{4}$ as a reference. Nitrogen adsorption-desorption isotherms at $77 \mathrm{~K}$ were performed using a Micromeritics ASAP2020M $+\mathrm{C}$ volumetric adsorption analyzer after the samples were degassed at $180^{\circ} \mathrm{C}$ overnight. The total organic carbon (TOC) of solutions was determined using a TOC analyzer (Analytik Jena multi N/C 3100 TOC, Germany).

\subsection{Photocatalytic measurement}

The photocatalytic activities of the $\mathrm{BiOCl}$ nanoplates were evaluated by batch experiments, using a LIMX-VII apparatus manufactured by Bylabo Precision Instruments (Xi'an, China), equipping with a $400 \mathrm{~W}$ halogen lamp (Institute for Electric Light Sources, China) and a $420 \mathrm{~nm}$ cutoff filter glass. Prior to the irradiation, the suspension containing $20 \mathrm{mg}$ photocatalyst and $40 \mathrm{~mL} 20 \mathrm{mg} / \mathrm{L} \mathrm{RhB}$ was kept stirring in dark for $1 \mathrm{~h}$ to reach the adsorption-desorption equilibrium. The $\mathrm{pH}$ value of $\mathrm{RhB}$ solution was unregulated unless otherwise stated. During irradiation, $3.5 \mathrm{~mL}$ aliquots were sampled at given time intervals, diluted two times, centrifuged to remove suspended photocatalyst particles, and then analyzed by T6 UV-Vis spectrophotometer (Beijing Purkinje General Instruments, China) at the maximum absorption band $554 \mathrm{~nm}$. The photocatalytic performance of Degussa $\mathrm{TiO}_{2}$ was also performed as a reference. Decrease of $\mathrm{RhB}$ concentration by adsorption after $1 \mathrm{~h}$ over all tested samples is collected in Supporting Information Tab. S1.

Photocatalytic measurement of sample BOC20-24 was also conducted over oxalic acid, a nonaromatic compound. A similar procedure was adopted except that $5 \times 10^{-3} \mathrm{M}$ oxalic acid was used in place of $20 \mathrm{mg} \mathrm{L}^{-1} \mathrm{RhB}$. After separation of catalyst nanoparticles, the supernatant was analyzed by TOC analyzer.

In order to understand the photocatalysis mechanism in detail, active species capture experiments were carried out to explore effects of hydroxyl radicals $\left({ }^{\circ} \mathrm{OH}\right)$ and superoxide radicals $\left({ }^{\circ} \mathrm{O}_{2}^{-}\right)$on

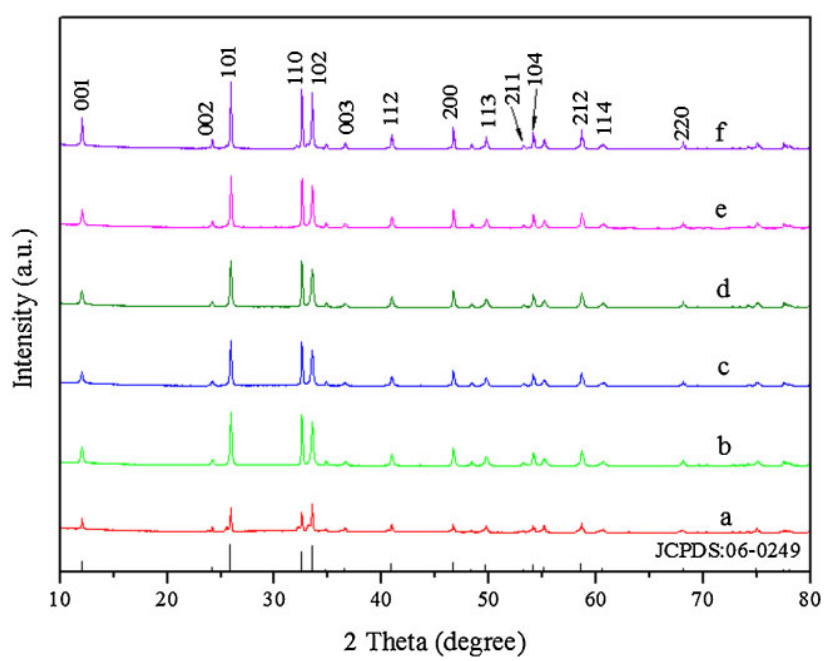

Figure 1. XRD patterns of the $\mathrm{BiOCl}$ samples: (a) BOC0-6 nanoflakes; nanoplates of (b) BOC5-6 and (c) BOC10-6; (d) flower-like BOC20-6; (e) flower-like BOC20-24 and (f) BOC30-6. 
photocatalytic efficiency by addition of $1.0 \mathrm{mM}$ IPA (a quencher of ${ }^{\circ} \mathrm{OH}$ ) and $\mathrm{BQ}$ (a quencher of ${ }^{\circ} \mathrm{O}_{2}^{-}$) into reaction systems, respectively [24-26]. The existence of ${ }^{\circ} \mathrm{OH}$ was also detected by a terephthalic acid fluorescence method on a Shimadzu RF-5301PC spectrofluorophotometer [27]. The employed excitation light in recording fluorescence spectra was $320 \mathrm{~nm} . \mathrm{N}_{2}$ bubbling experiment was also checked through expelling dissolved oxygen with bubbling $\mathrm{N}_{2}$ gas.

\section{Results and discussion}

\subsection{Characterization of nanostructured $\mathrm{BiOCl}$ samples}

The crystallinity and purity of as-synthesized BiOCl samples obtained with different PVA concentrations and hydrothermal time were examined by XRD patterns, depicted in Fig. 1. All samples can be indexed to a tetragonal matlockite $\mathrm{BiOCl}$ phase with lattice constants of $a=b=3.891 \AA$, and $c=7.369 \AA$, according to the standard card JCPDS 06-0249. From all XRD patterns, no peak around $19.1^{\circ}$ is observed, indicating the PVA polymer was completely removed after calcination [28]. Other impurities peaks are undetectable, revealing the high-purity and single-phase of as-synthesized BiOCl samples. The diffraction peaks of samples $\mathbf{b}-\mathbf{f}$ synthesized with the aid of PVA are narrow and high intensity in comparison with the sample a synthesized in the absence of PVA, indicating that addition of PVA and extension of hydrothermal time may somewhat improve the crystallinity. It is general that PVA are beneficial to stabilize metal ions via chelating and entrapment of metal ions into the polymeric network, which facilitates slow formation rate of nuclei and thus enhancement of crystallinity.

As shown in Supporting Information Fig. S1 and Tab. S2, the synthesized sample consists of elements $\mathrm{Bi}, \mathrm{O}$, and $\mathrm{Cl}$ with an atomic ratio of approximately 1:1:1 by EDX, which is quite consistent with the given formula BiOCl. Figure 2 shows the SEM images of BiOCl samples prepared with or without PVA. Without PVA, BOC0-6 nanoflakes were obtained with an average diameter of 1-2 $\mu \mathrm{m}$ and the thickness of $0.2 \mu \mathrm{m}$. These nanoflakes have a flat surface and each nanoflake is unattached and not interlaced with others. With the increase of PVA concentration and prolonging the hydrothermal time, BOC nanoplates were formed and further interlaced with each other with the assistance of PVA to assemble forward to 3D hierarchical flower-like structures, like samples BOC20-6 and BOC20-24. These nanoplates are not very regular in shape or uniform in size, but the thickness is reduced to nearly $0.1 \mu \mathrm{m}$ after introducing PVA. An excess amount of PVA might, on the contrary, prevent the connection and assembly of nanoplates, and these nanoplates could be closely stacked after calcinations, thus damaging the 3D hierarchical flower-like structure, like the sample BOC30-6.

UV-Vis diffuse reflectance spectra were measured to characterize the optical properties of the BiOCl samples. As can be seen in Fig. 3, the $\mathrm{BiOCl}$ nanoplates prepared with PVA exhibit an obvious red-shift of the absorption edges to visible light region in comparison with the BOC0-6 nanoflakes, indicating the possible photocatalytic ability under visible light irradiation. As reported, the structure, morphology and size of the semiconductor nanostructures have an important impact on relevant optical properties and offer a way tuning the band gap [29]. All BiOCl samples show a narrower band gap and the calculated band gap energies are lower than the theoretic value $(3.46 \mathrm{eV})$ [30]. Both hierarchical flower-like samples BOC20-6 and BOC20-24 show relatively low band gap energies as 3.26 and $3.37 \mathrm{eV}$, respectively.

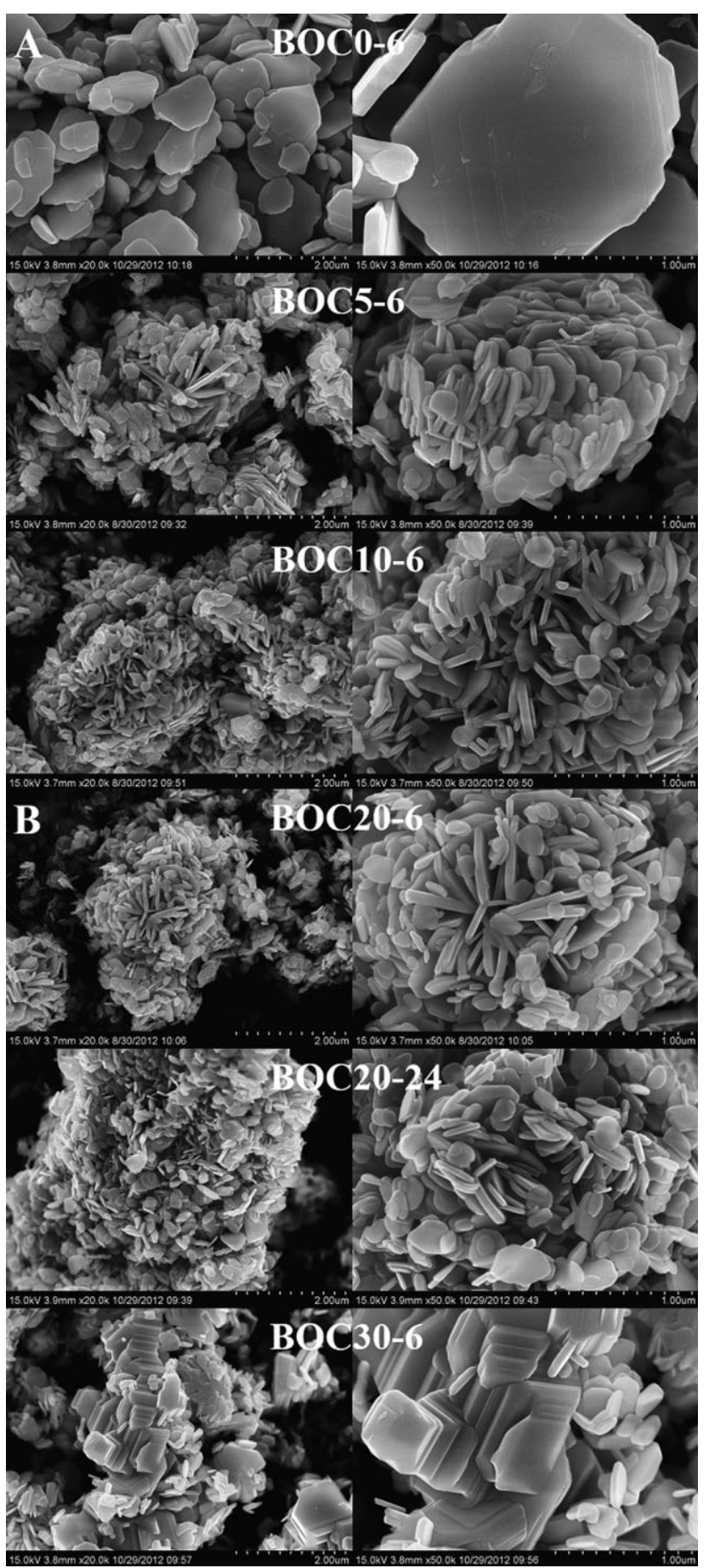

Figure 2. SEM patterns of $\mathrm{BiOCl}$ samples.

To further confirm the microstructure of synthesized samples, three typical samples BOC0-6 nanoflakes, flower-like BOC20-6, and BOC20-24 nanoplates together with Degussa $\mathrm{TiO}_{2}$ were exposed to the nitrogen adsorption-desorption analysis (Supporting Information Fig. S2). It is observed that adsorption ability of these three samples were in a sequence as BOC20-6 > BOC20-24 $>$ BOC0-6 under an identical condition. Likely, The BET specific surface areas calculated from the $\mathrm{N}_{2}$ isotherms are $5.85 \mathrm{~m}^{2} / \mathrm{g}$ (flower-like BOC20-6 nanoplates), $3.32 \mathrm{~m}^{2} / \mathrm{g}$ (flower-like BOC20-24 nanoplates), and $0.57 \mathrm{~m}^{2} / \mathrm{g}$ (BOC0-6 


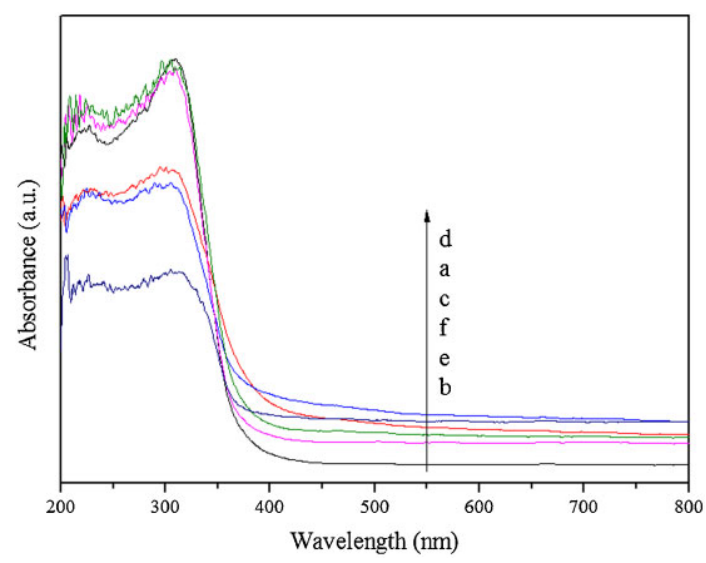

Figure 3. UV-Vis diffuse reflectance spectra of (a) BOC0-6 nanoflakes; nanoplates of (b) BOC5-6 and (c) BOC10-6; (d) flower-like BOC20-6; (e) flower-like BOC20-24, and (f) BOC30-6.

nanoflakes), respectively, $<55.5 \mathrm{~m}^{2} / \mathrm{g}$ (Degussa $\mathrm{TiO}_{2}$ ). The specific surface area value of flower-like BOC20-6 nanoplates is around ten times larger than that of BOC0-6 nanoflakes. It can be suspected that hierarchical flower-like BOC20-6 nanoplates may be the best candidate for the photocatalytic performance in this $\mathrm{BiOCl}$ series, owning to the large specific surface area and abundant reactive sites available [31].

\subsection{Formation of $\mathrm{BiOCl}$ nanostructured samples}

$\mathrm{BiOCl}$ can easily form the 2D laminar structure through the strong intra-layer bonding and the weak interlayer van der Waals interaction [32]. Suitable templates exert a remarkable effect on fabrication of hierarchical structures [33]. In this study, the polymeric PVA was proposed to play two main roles: (1) a good capping agent for adjusting the relative activity of the $\mathrm{Bi}^{3+}$ cation and kinetically retarding the grain growth [8]; (2) selective adsorption on the facets of $\mathrm{BiOCl}$ grains to promote the attachment of $\mathrm{BiOCl}$ nanoplates [6]. PVA coordinated with metal ions to form alkoxides, which significantly decreased the free $\mathrm{Bi}^{3+}$ concentration in solution and thus resulted into a relatively slow formation rate of the $\mathrm{BiOCl}$ nuclei when the saturated $\mathrm{NaCl}$ was added. The adsorbed PVA on the surface can stabilize the nanograins against further crystal growth, caused by a variation of the solid-liquid interface energy [10]. It is beneficial to form high-quality crystals. In addition, PVA can function as a directing agent in the formation of hierarchical flower-like $\mathrm{BiOCl}$ nanoplates due to its long chain and polyhydroxyl [6]. With the soft long carbon backbone and polyhdroxyl groups, the formed crystallines were stabilized into the polymeric network and interlaced with each other with the assistance of PVA. Therefore, these generated nanoplates further acted as building blocks in aggregation-based process and assembled into flower-like hierarchitecture $\mathrm{BiOCl}$ after removal of PVA by calcination. However, addition of excess amount of PVA might contrarily destroy the hierarchical flower-like structures through interrupting the connection of nanoplates, as stated above.

\subsection{Decomposition measurements}

The degradation of RhB was selected to evaluate the photocatalytic performance of $\mathrm{BiOCl}$ samples under visible light illumination $(\lambda>420 \mathrm{~nm})$ at room temperature. Figure 4 shows the temporal evolution of the absorption spectra of the RhB solution catalyzed by the flower-like BOC20-6 nanoplates. The adsorption peak centered at 250 and $300 \mathrm{~nm}$ gradually diminishes with the irradiation time extension and completely disappears after $120 \mathrm{~min}$, implying the destruction of the conjugated structure of $\operatorname{RhB}[34,35]$. The stepwise hypsochromic shift of the main absorption peak from 554 to $496 \mathrm{~nm}$ can be observed, assigning to the step-by-step deethylation of RhB [36]. In order to simplify the analysis, the characteristic absorption peak at $554 \mathrm{~nm}$ was employed to evaluate the photocatalytic degradation.

Figure $5 \mathrm{~A}$ shows that the variations of $\mathrm{RhB}$ concentration $\left(C / C_{0}\right)$ versus irradiation time over $\mathrm{BiOCl}$ samples. Direct photolysis of dye $\mathrm{RhB}$ is neglectable in this study. When Degussa $\mathrm{TiO}_{2}$ was added, merely $10 \% \mathrm{RhB}$ was removed after $120 \mathrm{~min}$ via a photosensitization pathway [35]. In contrast, the concentration of $\mathrm{RhB}$ in the presence of as-synthesized samples obviously decreased under the exposure to the visible light irradiation, indicating the high photocatalytic degradation capability. Among all $\mathrm{BiOCl}$ samples, hierarchical flowerlike BOC20-6 nanoplates achieved the highest photocatalytic performance. Over $99 \%$ of $\mathrm{RhB}$ could be consumed over sample BOC20-6 after illumination for $60 \mathrm{~min}$, while only $45 \%$ of RhB were removed over sample BOC0-6. In addition, another hierarchical flower-like sample BOC20-24 nanoplate exhibited comparable removal ability to BOC20-6, as can be seen in Fig. 5A. The variation of TOC was traced to determine the "real" mineralization of RhB. Figure 5B shows the time independence of the TOC results using BOC20-6 nanoplates as a photocatalyst. The TOC decrease of $45 \%$ was gained after $2 \mathrm{~h}$, much lower than the value of $100 \%$ obtained by UVVis absorption analysis. This is mainly attributed to the complex multi-step of complete mineralization of $\mathrm{RhB}$ and only the TOC in the solution taken into account, as reported in the literature [36].

In this work, synthesized BOCl samples could only absorb the UV light $(\lambda<390 \mathrm{~nm})$ on the basis of its UV-Vis diffuse reflectance edges. In other words, BiOCl samples could not be excited to generate the electrons and holes by visible light $(\lambda>400 \mathrm{~nm})$ because of its wide band gap. Therefore, $\mathrm{RhB}$ degradation by $\mathrm{BiOCl}$ nanostructures through photosensitization pathway was approvable. Photodegradation of nonaromatic compound oxalic acid by sample BOC20-24 was also tested under the same condition and no obvious decrease of

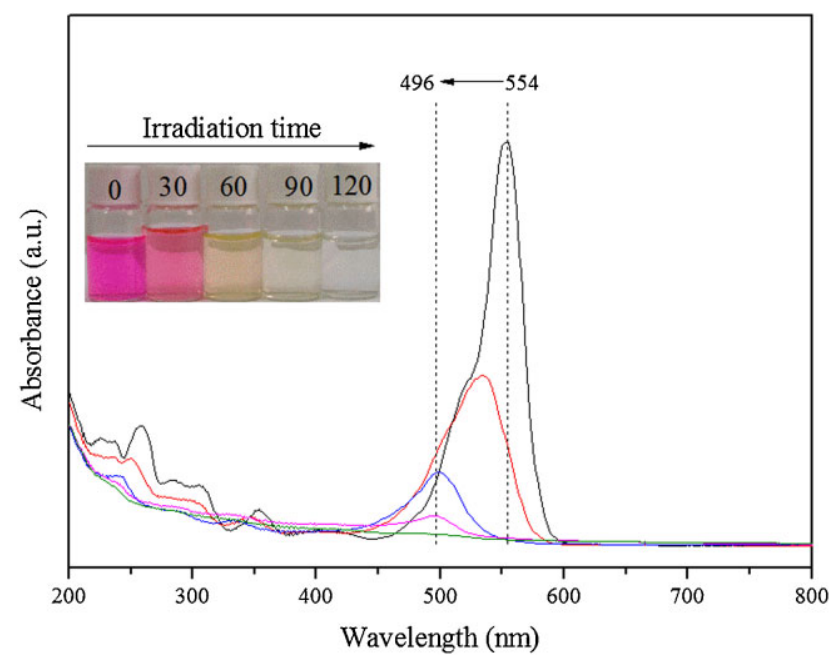

Figure 4. The temporal evolution of the absorption spectra of RhB over flower-like BOC20-6 nanoplates. 

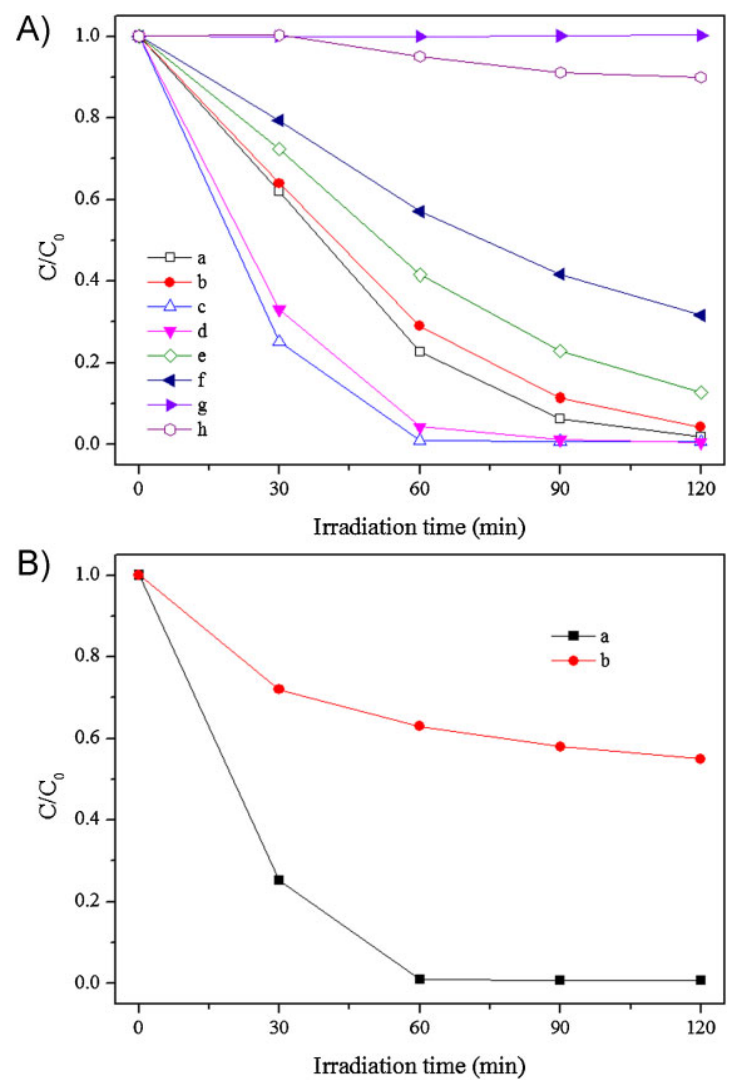

Figure 5. Photocatalytic activities of $\mathrm{BiOCl}$ series on RhB: (A) photocatalytic activities of (a) BOC0-6; (b) BOC10-6; (c) BOC20-6; (d) BOC2024; (e) BOC30-6; (f) BOC0-6; (g) Blank; (h) Degussa $\mathrm{TiO}_{2}$ by UV-Vis analysis. (B) Photocatalytic activity of BOC20-6 nanoplates by (a) UV-Vis analysis and (b) TOC analysis.

oxalic acid concentration further identified the photosensitization process when $\mathrm{BiOCl}$ samples were explored as photocatalysts, as can be seen in Supporting Information Fig. S3.

It is general that semiconductors with appropriate morphologies exert an important role for the efficient photocatalysis [9]. Based upon SEM observations, formation of 2D nanoplates from crystal grains and assembly of 2D nanoplates to 3D flower-like hierarchitecture with the aid of PVA generated channels, facilitating that reactants accessed the interior active sites from bulk solution and produced molecules departed the active sites $[37,38]$. Moreover, the hierarchical flower-like nanostructures allowed multiple scattering of visible light, resulting in greater light-harvesting capacities [39]. In addition, other factors like specific surface area can affect the photocatalysis as well. From the BET analysis, the hierarchical flower-like BOC20-6 nanoplates own the much larger specific surface area value than BOC0-6 nanoflakes, rendering sufficient active sites to expose the reactant molecules. As a result, 3D hierarchical flower-like $\mathrm{BiOCl}$ nanoplates showed the highest photocatalytic performance, possibly attributing to the intrinsic characteristic-layered structure, large specific surface area, and abundant reactive sites.

\subsection{Photocatalytic mechanism}

To understand the photocatalysis mechanism, roles of active species hydroxyl radicals $\left({ }^{\circ} \mathrm{OH}\right)$ and superoxide radicals $\left({ }^{\circ} \mathrm{O}_{2}^{-}\right)$were

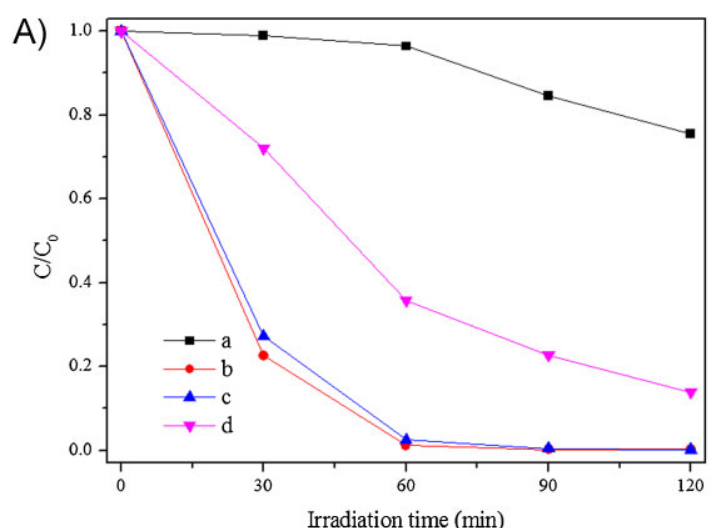

B)

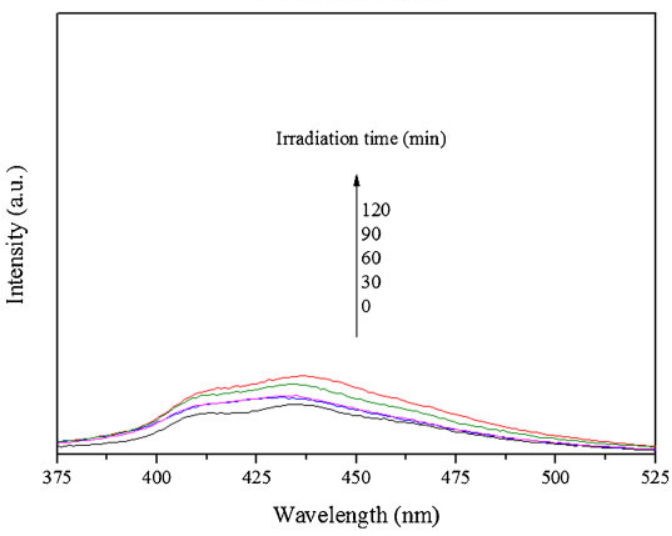

Figure 6. Active species capture experiment: (A) photodegradation efficiency of RhB on BOC20-24 nanoplates (a) BQ; (b) alone; (c) IPA; (d) $\mathrm{N}_{2}$ bubbling. (B) Fluorescence spectra of $\mathrm{TAOH}$ solution generated by BOC20-24 sample.

investigated by IPA (a quencher of ${ }^{\circ} \mathrm{OH}$ ) and $\mathrm{BQ}$ (a quencher of $\cdot \mathrm{O}_{2}^{-}$). As shown in Fig. 6A, the photodegradation efficiency of RhB kept almost same after introduction of IPA, indicating that the ${ }^{\circ} \mathrm{OH}$ was not the main reactive radicals. However, the removal efficiency of $\mathrm{RhB}$ over $\mathrm{BiOCl}$ decreased obviously from 100 to $25 \%$ when $1 \mathrm{mM}$ BQ was added, confirming that ${ }^{\circ} \mathrm{O}_{2}^{-}$radicals were mainly responsible for the photodegradation of RhB. $\mathrm{N}_{2}$ bubbling was applied to expel oxygen molecules dissolved in the aqueous, which would decrease entrapment of photoinduced electrons by oxygen to form ${ }^{\circ} \mathrm{O}_{2}^{-}$radicals. The control experiments rendered low degradation efficiency. Photoluminescence (PL) technique was further employed to detect the hydroxyl radicals formed on the surface of photocatalysts in a semiquantitative manner by checking the fluorescent intensity of 2 hydroxyterephthalic acid at $425 \mathrm{~nm}$ [27]. In this study, no hydroxyl radicals can be detected in the photocatalytic system with BOC20-24 nanoplates in Fig. 6B, further implying that the oxidation of $\mathrm{RhB}$ dye in aqueous solution was not mainly caused by hydroxyl radicals, which is in good consistence with references [24, 40].

As a result, under visible light illumination, the synthesized BiOCl could not be exited directly. The adsorbed $\mathrm{RhB}$ molecules were excited to form excited state $\mathrm{RhB}^{*}$ and subsequently excited state $\mathrm{RhB}^{*}$ injected electrons to the conduction band of BiOCl. The electron injected was captured by the oxygen molecules adsorbed on the surface of $\mathrm{BiOCl}$ to generate radicals ${ }^{\circ} \mathrm{O}_{2}^{-}$and ${ }^{\circ} \mathrm{OH}$ [41]. These active radicals finally degraded excited state $\mathrm{RhB}^{*}$ to complete decolorization of aqueous, as can be seen in Fig. 7. 


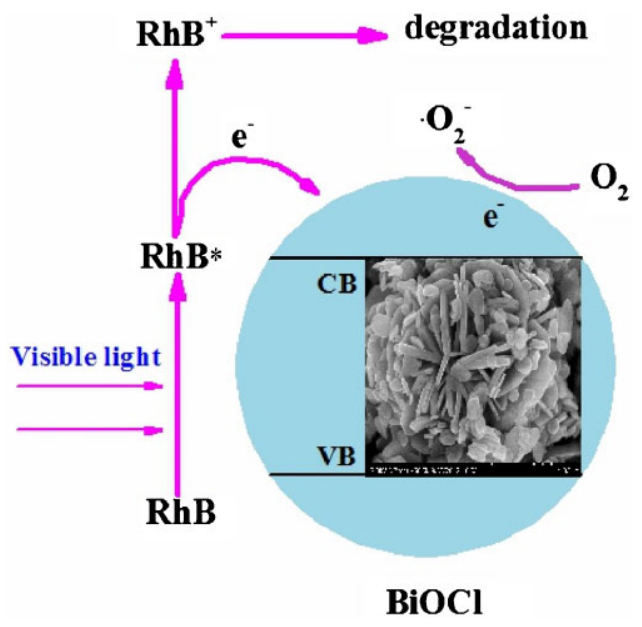

Figure 7. Possible photocatalytic mechanism for RhB by nanostructured $\mathrm{BiOCl}$ under visible light irradiation.

\section{Concluding remarks}

In summary, with the assistance of PVA, a series of hierarchical BiOCl nanoplates, including flower-like samples BOC20-6 and BOC20-24, were successfully synthesized by an one-pot hydrothermal process. It is found that addition of PVA and hydrothermal time played key roles in the formation of hierarchical BiOCl nanoplates. The photocatalytic activity of $\mathrm{BiOCl}$ series on the degradation of dye $\mathrm{RhB}$ were evaluated and hierarchical flower-like BiOCl nanoplates exhibited the highest photocatalytic activity comparing to other $\mathrm{BiOCl}$ nanoplates, BOC0-6 nanoflakes and Degussa $\mathrm{TiO}_{2}$, mainly attributing to the synergistic effect, including intrinsic characteristic-layered structure, large specific surface area, and abundant reactive sites. The photocatalysis mechanism test indicated that superoxide radicals $\left({ }^{\circ} \mathrm{O}_{2}^{-}\right)$were mainly responsible for the efficient photodegradation of $\mathrm{RhB}$. The present work developed new promising visible-light-driven photocatalysts and will potentially implement efficient applications for degrading organic pollutants.

\section{Acknowledgements}

We are grateful to the National Natural Science Foundation of China (Grant number 21207089, 41076040, and 20807036), the Innovation Program of Shanghai Municipal Education Commission (Grant number 11YZ113), the Special Research Fund in Shanghai Colleges and Universities to Select and Train Outstanding Young Teachers (Grant number slg10014), the project-sponsored by SRF for ROCS, SEM., and the "Chen Guang" project supported by Shanghai Municipal Education Commission and Shanghai Education Development Foundation (Grant number 11CG52) for financial support.

The authors have declared no conflict of interest.

\section{References}

[1] T. L. Thompson, J. T. Yates, Surface Science Studies of the Photoactivation of $\mathrm{TiO}_{2}$ : New Photochemical Processes, Chem. Rev. 2006, 106 (10), 4428-4453.

[2] M. R. Hoffmann, S. T. Martin, W. Choi, D. W. Bahnemann, Environmental Applications of Semiconductor Photocatalysis, Chem. Rev. 1995, 95 (1), 69-96.
[3] K. L. Zhang, C. M. Liu, F. Q. Huang, C. Zheng, W. D. Wang, Study of the Electronic Structure and Photocatalytic Activity of the BiOCl Photocatalyst, Appl. Catal., B 2006, 68 (3-4), 125-129.

[4] C. F. Guo, S. H. Cao, J. M. Zhang, H. Y. Tang, S. M. Guo, Y. Tian, Q. Liu, Topotactic Transformations of Superstructures: From Thin Films to Two-Dimensional Networks to Nested Two-Dimensional Networks, J. Am. Chem. Soc. 2011, 133 (21), 8211-8215.

[5] X. P. Lin, T. Huang, F. Q. Huang, W. D. Wang, J. L. Shi, Photocatalytic Activity of a Bi-Based Oxychloride $\mathrm{Bi}_{3} \mathrm{O}_{4} \mathrm{Cl}$, J. Phys. Chem. B 2006, 110 (48), 24629-24634.

[6] J. Y. Xiong, G. Cheng, G. F. Li, F. Qin, R. Chen, Well-Crystallized Square-Like 2D BiOCl Nanoplates: Mannitol-Assisted Hydrothermal Synthesis and Improved Visible-Light-Driven Photocatalytic Performance, RSC Adv. 2011, 1 (8), 1542-1553.

[7] X. P. Lin, T. Huang, F. Q. Huang, W. D. Wang, J. L. Shi, Photocatalytic Activity of a Bi-Based Oxychloride $\mathrm{Bi}_{4} \mathrm{NbO}_{8} \mathrm{Cl}$, J. Mater. Chem. 2007, 17 (20), 2145-2150.

[8] K. Zhang, J. Liang, S. Wang, J. Liu, K. X. Ren, X. Zheng, H. Luo, et al., BiOCl Sub-Microcrystals Induced by Citric Acid and Their High Photocatalytic Activities, Cryst. Growth Des. 2012, 12 (2), 793803.

[9] J. M. Song, C. J. Mao, H. L. Niu, Y. H. Shen, S. Y. Zhang, Hierarchical Structured Bismuth Oxychlorides: Self-assembly from Nanoplates to Nanoflowers via a Solvothermal Route and Their Photocatalytic Properties, CrystEngComm 2010, 12 (11), 3875-3881.

[10] Y. Lei, G. Wang, S. Song, W. Fan, H. Zhang, Synthesis, Characterization and Assembly of BiOCl Nanostructure and Their Photocatalytic Properties, CrystEngComm 2009, 11 (9), 1857-1862.

[11] F. Chen, H. Q. Liu, S. Bagwasi, X. X. Shen, J. L. Zhang, Photocatalytic Study of BiOCl for Degradation of Organic Pollutants under UV Irradiation, J. Photochem. Photobiol., A 2010, 215 (1), 76-80.

[12] J. Geng, W. H. Hou, Y. N. Lv, J. J. Zhu, H. Y. Chen, One-dimensional $\mathrm{BiPO}_{4}$ Nanorods and Two-dimensional BiOCl Lamellae: Fast Lowtemperature Sonochemical Synthesis, Characterization, and Growth Mechanism, Inorg. Chem. 2005, 44 (23), 8503-8509.

[13] J. M. Ma, X. D. Liu, J. B. Lian, X. C. Duan, W. J. Zheng, Ionothermal Synthesis of BiOCl Nanostructures via a Long-Chain Ionic Liquid Precursor Route, Cryst.Growth Des. 2010, 10 (6), 25222527.

[14] H. Deng, J. W. Wang, Q. Peng, X. Wang, Y. D. Li, Controlled Hydrothermal Synthesis of Bismuth Oxyhalide Nanobelts and Nanotubes, Chem. Eur. J. 2005, 11 (22), 6519-6524.

[15] C. Wang, C. Shao, Y. Liu, L. Zhang, Photocatalytic Properties BiOCl and $\mathrm{Bi}_{2} \mathrm{O}_{3}$ Nanofibers Prepared by Electrospinning, Scr. Mater. 2008, 59 (3), 332-335.

[16] Z. T. Deng, D. Chen, B. Peng, F. Q. Tang, From Bulk Metal Bi to Twodimensional Well-crystallized BiOX $(\mathrm{X}=\mathrm{Cl}, \mathrm{Br})$ Micro- and Nanostructures: Synthesis and Characterization, Cryst. Growth Des. 2008, 8 (8), 2995-3003.

[17] L. Q. Ye, L. Zan, L. H. Tian, T. Y. Peng, J. J. Zhang, The \{001\} FacetsDependent High Photoactivity of BiOCl Nanosheets, Chem. Commun. 2011, 47 (24), 6951-6953.

[18] Y. Y. Li, J. P. Liu, J. Jiang, J. G. Yu, UV-Resistant Superhydrophobic BiOCl Nanoflake Film by a Room-temperature Hydrolysis Process, Dalton Trans. 2011, 40 (25), 6632-6634.

[19] K. Zhang, D. Q. Zhang, J. Liu, K. X. Ren, H. Luo, Y. J. Peng, G. S. Li, X. B. Yu, A Novel Nanoreactor Framework of Iodine-incorporated BiOCl Core-Shell Structure: Enhanced Light-Harvesting System for Photocatalysis, CrystEngComm 2012, 14 (2), 700-707.

[20] L. Chen, S. F. Yin, R. Huang, Y. Zhou, S. L. Luo, C. T. Au, Facile Synthesis of BiOCl Nano-Flowers of Narrow Band Gap and Their Visible-Light-Induced Photocatalytic Property, Catal. Commun. 2012, 23, 54-57.

[21] L. P. Zhu, G. H. Liao, N. C. Bing, L. L. Wang, Y. Yang, H. Y. Xie, SelfAssembled 3D BiOCl Hierarchitectures: Tunable Synthesis and Characterization, CrystEngComm 2010, 12 (11), 3791-3796.

[22] T. Liu, Y. Xu, C. L. Zeng, Synthesis of $\mathrm{Bi}_{2} \mathrm{Fe}_{4} \mathrm{O}_{9}$ Via PVA Sol-Gel Route, Mater. Sci. Eng., B 2011, 176 (7), 535-539. 
[23] R. Jain, M. Mathur, S. Sikarwar, A. Mittal, Removal of the Hazardous Dye Rhodamine B through Photocatalytic and Adsorption Treatments, J. Environ. Manage. 2007, 85 (4), 956-964.

[24] L. Q. Ye, C. Q. Gong, J. Y. Liu, L. H. Tian, T. Y. Peng, K. J. Deng, L. Zan, $\mathrm{Bi}_{\mathrm{n}}(\mathrm{Tu})_{\mathrm{x}} \mathrm{Cl}_{3 \mathrm{n}}:$ A Novel Sensitizer and Its Enhancement of $\mathrm{BiOCl}$ Nanosheets' Photocatalytic Activity, J. Mater. Chem. 2012, 22 (17), 8354-8360.

[25] Y. N. Wang, K. J. Deng, L. Z. Zhang, Visible Light Photocatalysis of BiOI and Its Photocatalytic Activity Enhancement by in Situ Ionic Liquid Modification, J. Phys. Chem. C 2011, 115 (29), 1430014308.

[26] X. F. Hu, T. Mohamood, W. H. Ma, C. C. Chen, J. C. Zhao, Oxidative Decomposition of Rhodamine B Dye in the Presence of $\mathrm{VO}_{2}{ }^{+}$and/or $\mathrm{Pt}(\mathrm{IV})$ under Visible Light Irradiation: $N$-Deethylation, Chromophore Cleavage, and Mineralization, J. Phys. Chem. B 2006, 110 (51), 2601226018.

[27] X. S. Zhou, B. Jin, L. D. Li, F. Peng, H. J. Wang, H. Yu, Y. P. Fang, A Carbon Nitride/ $\mathrm{TiO}_{2}$ Nanotube Array Heterojunction Visible-Light Photocatalyst: Synthesis, Characterization, and Photoelectrochemical Properties, J. Mater. Chem. 2012, 22 (34), 17900-17905.

[28] R. C. L. Mooney, An X-Ray Study of the Structure of Polyvinyl Alcohol, J. Am. Chem. Soc. 1941, 63 (10), 2828-2832.

[29] G. Dukovic, F. Wang, D. H. Song, M. Y. Sfeir, T. F. Heinz, L. E. Brus, Structural Dependence of Excitonic Optical Transitions and BandGap Energies in Carbon Nanotubes, Nano Lett. 2005, 5 (11), 23142318.

[30] H. L. Peng, C. K. Chan, S. Meister, X. F. Zhang, Y. Cui, Shape Evolution of Layer-Structured Bismuth Oxychloride Nanostructures via Lowtemperature Chemical Vapor Transport, Chem. Mater. 2009, 21 (2), 247-2252.

[31] M.A. Fox, M. T. Dulay, Heterogeneous Photocatalysis, Chem. Rev. 1993 93 (1), 341-357.
[32] A. M. Kusainova, W. H. Zhou, J. T. Irvine, P. Lightfoot, Layered Intergrowth Phases $\mathrm{Bi}_{4} \mathrm{MO}_{8} \mathrm{X}(\mathrm{X}=\mathrm{Cl}, \mathrm{M}=\mathrm{Ta}$ and $\mathrm{X}=\mathrm{Br}, \mathrm{M}=\mathrm{Ta}$ or $\mathrm{Nb}$ ): Structural and Electrophysical Characterization, J. Solid State Chem. 2002, 166 (1), 148-157.

[33] Y. G. Sun, B. Gates, B. Mayers, Y. N. Xia, Crystalline Silver Nanowires by Soft Solution Processing, Nano Lett. 2002, 2 (2), 165-168.

[34] C. Zhang, Y. F. Zhu, Synthesis of Square $\mathrm{Bi}_{2} \mathrm{WO}_{6}$ Nanoplates as Highactivity Visible-Light-Driven Photocatalysts, Chem. Mater. 2005, 17 (31), 3537-3545.

[35] J. Fu, Y. Tian, B. Chang, F. Xi, X. Dong, BiOBr-Carbon Nitride Heterojunctions: Synthesis, Enhanced Activity and Photocatalytic Mechanism, J. Mater. Chem. 2012, 22 (39), 21159-21166.

[36] W. Xiong, Q. D. Zhao, X. Y. Li, D. K. Zhang, One-step Synthesis of Flower-Like Ag/AgCl/BiOCl Composite with Enhanced Visible-Light Photocatalytic Activity, Catal. Commun. 2011, 16 (8), 229-233.

[37] J. G. Yu, Y. R. Su, B. Cheng, Template-Free Fabrication and Enhanced Photocatalytic Activity of Hierarchical Macro-/Mesoporous Titania, Adv. Funct. Mater. 2007, 17 (12), 1984-1990.

[38] J. G. Yu, L. J. Zhang, B. Cheng, Y. R. Su, Hydrothermal Preparation and Photocatalytic Activity of Hierarchically Sponge-Like Macro-/Mesoporous Titania, J. Phys. Chem. C 2007, 111 (28), 10582-10589.

[39] J. X. Xia, S. Yin, H. M. Li, H. Xu, L. Xu, Y. G. Xu, Improved Visible Light Photocatalytic Activity of Sphere-like BiOBr Hollow and Porous Structures Synthesized via a Reactable Ionic Liquid, Dalton Trans. 2011, 40 (19), 5249-5258.

[40] L. Q. Ye, J. Y. Liu, C. Q. Gong, L. H. Tian, T. Y. Peng, L. Zan, Two Different Roles of Metallic Ag on $\mathrm{Ag} / \mathrm{AgX} / \mathrm{BiOX}(\mathrm{X}=\mathrm{Cl}, \mathrm{Br})$ Visible Light Photocatalysts: Surface Plasmon Resonance and Z-Scheme Bridge, ACS Catal. 2012, 2 (8), 1677-1683.

[41] C. Chen, W. Ma, J. Zhao, Semiconductor-Mediated Photodegradation of Pollutants under Visible-Light Irradiation, Chem. Soc. Rev. 2010, 39 (11), 4206-4219. 